\title{
UM-CIRHTWebinar Series
}

\section{Framing a Research Question}

Berhanu Gebremeskel MD MPH

Sr. Project Manager, UM-CIRHT

Michigan Medicine 
"He who does not know what he is looking for will not lay hold of what he has found when he gets it"

Claude Bernard 


\section{UM-CIRHT's Research Overarching Goal}

- Inspire research culture at individual and institutional level 


\section{UM-CIRHT Framework: Research Life Cycle}

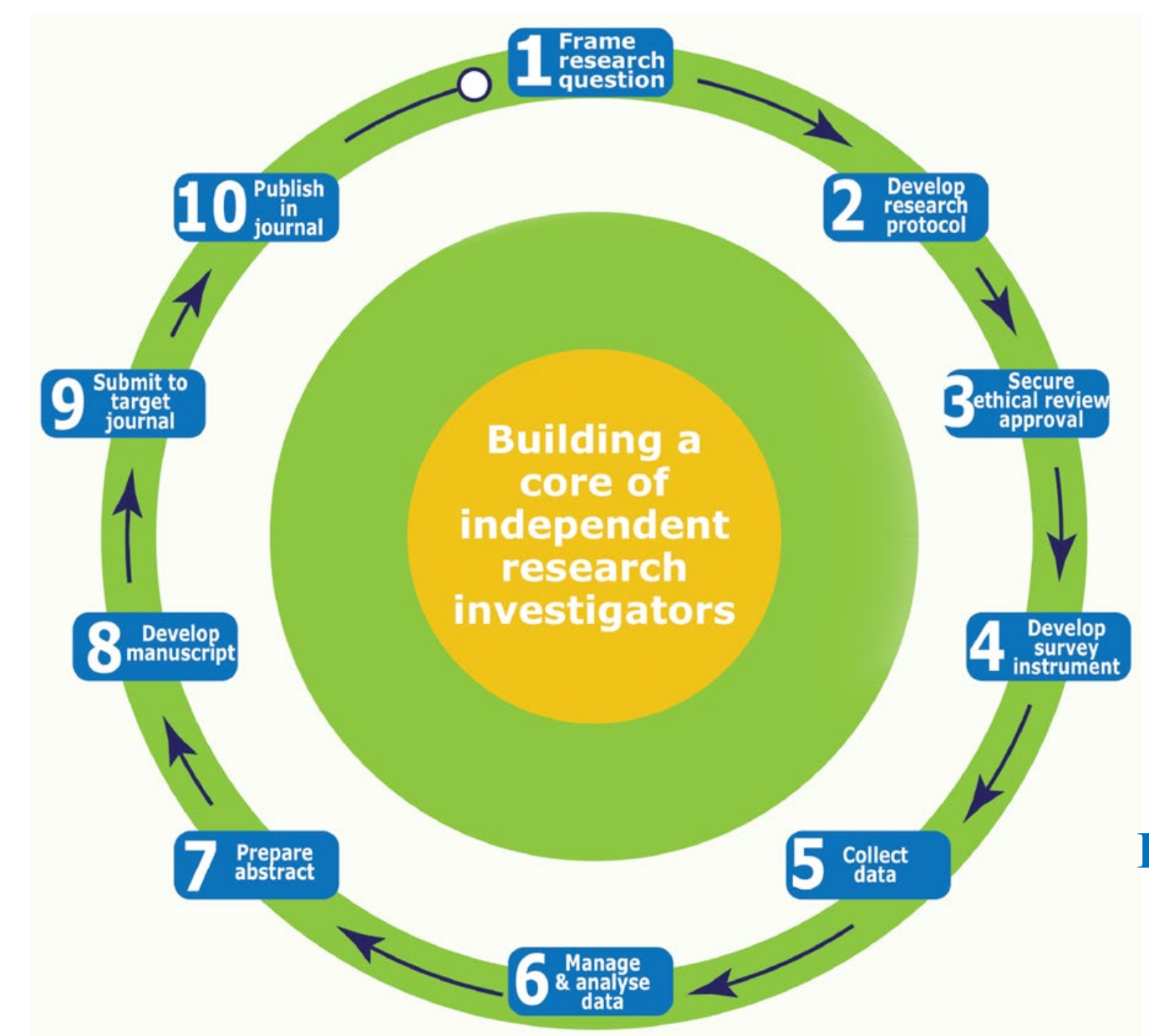

Beza et al, 2018. The UM-CIRHT Framework 


\section{Research Process}

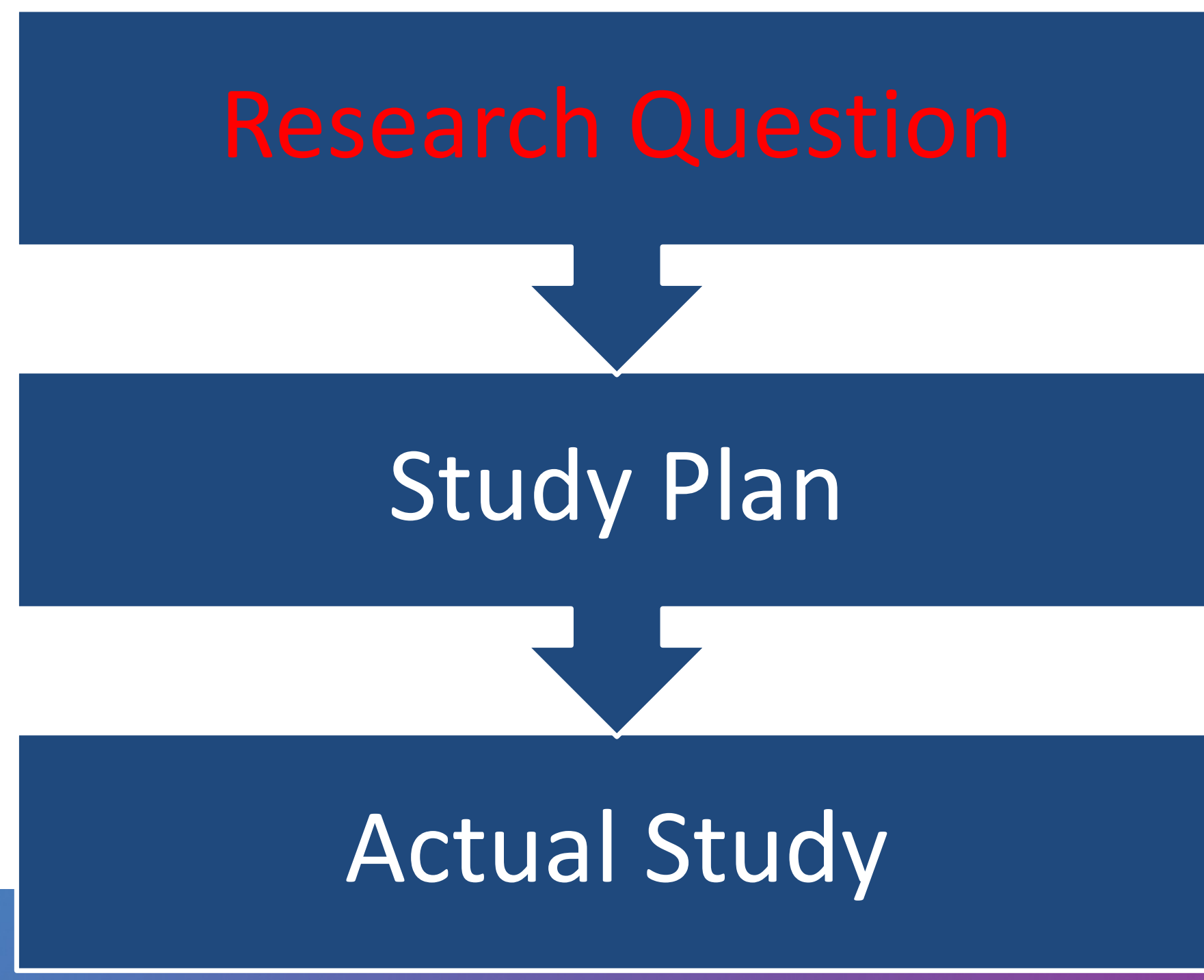




\section{Webinar Objectives}

- To illustrate the importance of framing a good research question

- Ask useful, answerable, valid question

-Introduce two useful frameworks for evaluating and refining a research question

-Through this endeavor:

- Help investigators develop valid and feasible study plan

- IRB Application

- Grant Application

-This webinar is not about:

- Study design

- Details of statistics 


\section{Outline}

-What is research?

-What is the research question?

-Why frame a research question?

- Sources of research questions

- Characteristics of a good research question

- FINER criteria

-Roadmap for framing a Research Question

- PICO(T) framework

-Examples

-Resources

- CIRHT guide

- NIH RePORT 


\section{What is Research?}

"Research means a systematic investigation, including research development, testing and evaluation, designed to develop or contribute to generalizable knowledge" (45 CFR 46)

- Clinical research vs. clinical practice [distinction very important]

- Activity designed to

- Test hypothesis

- Permit conclusions, thereby:

- Develop or contribute to generalizable knowledge (theories, principles, statement of relationships)

- Interventions designed solely to enhance well-being of individual patient or client and have reasonable expectation of success, purpose to provide diagnosis, preventive treatment or therapy to particular individuals 


\section{What is a Research Question?}

- "The uncertainty that the investigator wants to resolve by performing his/her own study"*

-The objective /aim of the study

- NIH Specific aims section

-Forms the foundation of the study

-Action oriented

- The research question will answer the question

-Examples

- "Is life worth living?" 


\section{What is a Research Question?}

- The boundary between current knowledge and ignorance -Researchable questions come from finding the 'cutting edge' of knowledge for a problem with which you are familiar* 


\section{Why Frame a Research Question?}

- The challenge in finding a research question is defining an important one that can be transformed into a feasible and valid study plan.

-Forms the basis of the study plan
TABLE 1.1 ANATOMY OF RESEARCH: THE STUDY PLAN

\begin{tabular}{ll}
\hline DESIGN COMPONENTS & PURPOSE \\
\hline Research questions & What questions will the study address? \\
\hline Background and significance & Why are these questions important? \\
\hline $\begin{array}{l}\text { Design } \\
\text { Time frame }\end{array}$ & How is the study structured? \\
Epidemiologic design & Who are the subjects and how will they be selected? \\
\hline $\begin{array}{l}\text { Subjects } \\
\text { Selection criteria }\end{array}$ \\
$\begin{array}{l}\text { Sampling design } \\
\text { Variables }\end{array}$ \\
$\begin{array}{l}\text { Predictor variables } \\
\text { Confounding variables } \\
\text { Outcome variables }\end{array}$ \\
$\begin{array}{l}\text { Statistical issues } \\
\text { Hypotheses } \\
\text { Sample size } \\
\text { Analytic approach }\end{array}$
\end{tabular}




\section{Writing the Researchable Question}

-Challenging and difficult step in the research process

- Yet: one of the most critical aspects

-A clear and carefully and thoughtfully constructed research question

- Helps guide the research project to hit its scholarly target

- Research is only as good as the research question and plan on which it is based

-Determines the success of a research process

- Should drive the development of a research protocol

-Research interest $\neq$ Research Question 


\section{Why Most Published Research Findings Are}

False

Open access, freely available online

Essay

\section{Why Most Published Research Findings}

\section{Are False \\ John P. A. loannidis}

Summary
There is increasing concern that most
current published research findings are
false. The probability that a research claim
is true may depend on study power and
bias, the number of other studies on the
same question, and, importantly, the ratio
of true to no relationships among the
relationships probed in each scientific
field. In this framework, a research finding
is less likely to be true when the studies
conducted in a field are smaller; when
effect sizes are smaller; when there is a
greater number and lesser preselection
of tested relationships; where there is
greater flexibility in designs, definitions,
outcomes, and analytical modes; when
there is greater financial and other
interest and preiudice; and when more

factors that influence this problem and is characteristic of the field and can some corollaries thereof. vary a lot depending on whether the

Model - _. - . field targets highly likely relationships

Positi Moreover, for many current scientific

Several
pointe

rate of often be simply accurate measures of th is a con prevailing bias. In this essay, I discuss the conclu implications of these problems for the the bas implications of these problems for the formal
for a $\mathrm{p}-\mathrm{c}$
conduct and interpretation of research.

and summarized by $p$-values, but, reflects the power $1-\beta$ (one minus

and summanzed by $p$-values, but,

unfortunately, there is a widespread notion that medical research articles 


\title{
Refining the Research Question
}

\section{General Research Topic}

Focus down the Question

\author{
Develop \\ Hypothesis \\ Specific




\section{Sources of Research Questions}

- Observations in clinical practice

- Your own research

-Quality improvement activities

-Mentors

-Colleagues

-Scientific conferences

-Scientific literature

-Use your imagination!!! 


\section{Characteristics of a Good Research Question:}

FINER Criteria 


\section{Characteristics of a Good Research Question: FINER Criteria}

TABLE 2.1 FINER CRITERIA FOR A GOOD RESEARCH QUESTION

AND STUDY PLAN

Feasible

Adequate number of subjects

Adequate technical expertise

Affordable in time and money

Manageable in scope

Fundable

Interesting

Getting the answer intrigues the investigator and her colleagues

Novel

Provides new findings

Confirms, refutes, or extends previous findings

May lead to innovations in concepts of health and disease, medical practice, or methodologies

for research

Ethical

A study that the institutional review board will approve

Hulley SB, Designing clinical research. LWW; 2013.

Relevant

Likely to have significant impacts on scientific knowledge, clinical practice, or health policy 


\section{Feasible}

-Adequate \# study subjects

-Appropriate/adequate technical expertise

-Within scope

-Adequate funding 


\section{Interesting}

- Getting at the truth of the matter is interesting

- Provides the necessary motivation/ intensity of effort for the research process

-Interesting to the researcher

- Others should also find it interesting

- Mentors

- Outside experts

- Funders 


\section{Novel}

-A good clinical research question contributes to new knowledge -Innovative: new

- Concepts

- Methods/approaches

- Interventions

-But: need not be totally original

- Replication: confirmatory

- Other population 


\section{Ethical}

-Must be ethical

-The first requirement of ethical research: research question must be worth asking: must have

- potential social, scientific, or clinical value

-If unacceptable risks/invasion of privacy:

- Other ways of answering the RQ

-If uncertain:

- Discuss with IRB reps early on

-Validity is an essential element of ethical research 


\section{Research must be ethical!}

-Valuable research question ethically requires validity and rigor in research design and implementation to produce valid, reliable, interpretable, and generalizable results

-Poorly designed research:

- with an inappropriate design

- inadequate power

- insufficient or sloppy data, or

- inappropriate or unfeasible methods is

- harmful because human and material resources are wastgin and individuals are exposed to risk for no benefit 


\section{Relevant}

-Significance

- Importance of the problem

- How the project will improve scientific knowledge

- How the result will change

- Concepts

- Methods

- Clinical practice 


\section{So What?}

Should pass the "So what? Test!

Will the research make a difference? 
Roadmap for Framing a Research Question: $\mathrm{PICO}(\mathrm{T})$ Framework 


\section{PICO(T) Framework}

-In order to develop and refine research ideas: useful techniques:

- Mind mapping

- Brainstorming

- Focus groups

-Very useful approach to transform research idea into a research question : PICOT format 


\section{PICO(T) Framework}

-P-Population[ patient/problem]

-I-Intervention [exposure, diagnostic test, prognostic factor]

-C-Comparison [if relevant']

-O-Outcome

-T-Timeframe 


\section{PICO(T) approach: Examples}

\section{Original Question}

I. What is the best way to prevent and manage spasticity in $\mathrm{TBI}$ patients

2. Why should we fund physiotherapy for patients following discharge from $\mathrm{SCl}$ rehab?

3. Why did you do a CT instead of an MRI for this TBI patient?

\section{PICO(T) format}

I. In patients with severe TBI, what is the effect of casting on spasticity, compared with pharmacological management $(R x)$

2. In patients following $\mathrm{SCl}$ rehab, what is the effect of community-based physiotherapy on functional status, compared with standard care? (DX)

3. In patients with suspected TBI, what is the diagnostic value of $\mathrm{CT}$, compared with MRI? (DX) 


\section{Another example}

contraception in the United States [13]. However, there is little information about effective strategies in Ethiopia and other LMICs. In Ethiopia contraception training traditionally indudes lectures during Obstetric and Gynecology (Ob-Gyn) clerkship but without specific skill-focuse FP exposure. Cognizant of this gap, competencies for all methods of reversible contraceptives were integrated into the medical school curriculum at St. Paul's Hospital Millennium Medical College (SPHMMC), Addis Ababa, in 2012, in collaboration with the Department of Ob-Gyn at the University of Michigan. Following this, the Center for International Reproductive Health Training (CIRHT) was established at the University of Michigan with the goal of decreasing matemal morbidity and mortality through ffective preservice training of medical students in Frta ity through efective preser. abortion. The University of Michigan is providing support for the integration program through various faculty development initiatives in thiopia.

The objective of this study was to assess if structured integration of comprehensive FP training into a medical school curnculum improves FP knowledge and skills of interns. We also investigated potential factors associated with knowledge and skills (demographics, selfassessed competencies and reported number of procedures). We hypothesized that interns from the intervention site with the structured integrated curriculum (SPHMMC) have higher knowledge and skill scores compared to four control sites with conventional curriculum.

\section{Material and methods}

This study conducted in five Ethiopian medical schools, combared the intervention school that imblemented a structured FP cur-

\subsection{Intervention site}

The structured integrated FP training at SPHMMC involved five FP didactic sessions during third year of medical school (three 1-h lectures, one tutorial and one seminar) and five simulation sessions during the fourth year to practice FP and abortion procedures [implant and IUD insertion and removal and manual vacuum aspiration (MVA)] under $\mathrm{Ob}$ Gyn faculty supervision. After a fourth-year qualifying exam, students complete a 1-year rotating internship before graduation. At the intervention site, interns had service rotations at SPHMMC's FP clinic for week within their 10 -week $O b$ - $y$ n rotation performing supervised week with their 10-week Ob-Gyn rotation, performing supervised  ing coverage of all essential content and minimizing any variability in the training. The faculty supervise the intems when doing LARC procedures. This paper reports $\mathrm{P}$ outcomes and does not present the result of the comprehensive abortion care training

\subsection{Control sites}

The four control medical schools have existed for at least 10 years. Their Ob-Gyn curriculum comprised $3 \mathrm{~h}$ of lectures on FP and comprehensive abortion care topics. There were no FP skill teaching in simulated or clinical settings, and no emphasis on students rotating through FP clinics during their internship. However, interns were expected to perform supervised MVAs in the management of patients with incomplete abortion during the gynecology rotation. They were not expected to place LARC devices or participate in family planning rotations during their internship year (Fig. 1)

Gebremeskel BG, Structured integration . Contraception 2018. 


\section{Examples of Good Research Questions}

-Funded Research Projects from the Contraception Research Branch (CRB) of the NICHD

- (2008-2019) EUNICE KENNEDY SHRIVER NATIONAL INSTITUTE OF CHILD HEALTH \& HUMAN DEVELOPMENT

-https://www.nichd.nih.gov/about/org/der/branches/crb/projects?f $y=2008$ 


\section{Examples of Good Research Questions}

I. Understanding the lactational amenorrhea method of contraception for mothers of preterm and term infants

I. to compare amenorrhea rates, duration of lactation, and resumption of sexual activity among postpartum women who are breastfeeding versus expressing milk after term and preterm delivery through a prospective observational study following women through 6 months postpartum

2. assessing the feasibility and acceptability of using home urinary luteinizing hormone test kits to detect ovulation in exclusively breastfeeding women

3. understand decision-making regarding contraception and infant feeding practices in mothers of preterm infants using a qualitative approach with semi-structured interviews

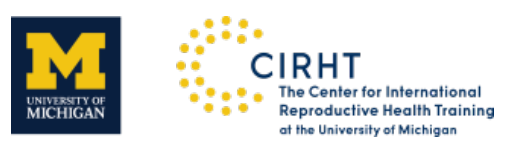




\section{Examples of Good Research Questions}

-RCT assessing pregnancy with intrauterine devices for emergency contraception

I. Our primary aim is to assess the efficacy of the LNG20 IUD vs. the copper T380 IUD for EC utilizing a non- inferiority designassessing the feasibility and acceptability of using home urinary luteinizing hormone test kits to detect ovulation in exclusively breastfeeding women

2. assess one-year continuation and unintended pregnancy rates in women initiating the LNG20 IUD or copper T380 IUD for EC and study the mechanisms of continuation related to satisfaction, pain, and bleeding over the first year of use 


\section{Examples of Good Research Questions}

- Contraceptive method and HIV risk

I. determine whether women randomized to DMPA have more or less frequent condomless vaginal sex than women randomized to the IUD or implant during trial follow-up by comparing vaginal PSA, a marker of recent semen exposure, between randomized contraceptive groups across multiple $\mathrm{ECHO}$ sites

2. evaluate whether women randomized to DMPA are more or less likely to misreport condomless sex than women randomized to the IUD or implant during trial follow-up by evaluating the concordance of selfreported condom use or abstinence during trial follow-up with PSA detection overall, and by randomized contraceptive group 


\section{Examples of Good Research Questions}

-A prospective cohort of HIV-infected Malawian women on Efavirenz initiating the levonorgestrel implant or the depot medroxyprogesterone acetate injectable

I. compare the typical-use pregnancy rates of the LNG implant versus the DMPA injectable in a prospective cohort of I,420 HIV+ women on EFV (7I0 initiating the LNG implant and 7I0 initiating DMPA

2. study 240 women in a 2:I nested case-control study of women from the cohort to determine if higher EFV concentrations in hair (a biomarker for EFV exposure) are associated LNG implant contraceptive failure. 80 women (cases) who become pregnant while using the LNG implant will be compared with I 60 LNG implant users who were not pregnant after the same duration of implant use (controls 


\section{Examples of Good Research Questions}

- Improving emergency contraceptive effectiveness in obese women

I. We hypothesize that increasing the dose of orally-dosed EC agents will normalize the pharmacokinetics resulting in the expected treatment effect (delay in follicle rupture) in obese women

I. , we plan to perform detailed pharmacokinetic and pharmacodynamic studies of ulipristal acetate- based EC in obese women and expand upon our preliminary findings of levonorgestrel-based EC. 


\section{Summary}

- Research question begins with an idea which is then transformed into a research question

-A research question: clear, focused, and concise statement that conveys the objectives of the research and its potential findings

-Should be expressed in a simple, straight-forward language 


\section{Before committing much time and effort to writing a proposal/carrying out a study:}

-Is the research question/study plan 'FINER'?

-Funder: priority to proposals that may have innovative and significant impacts on :

- Science and

- Health

-Early on: RQ into one page Study outline, specifically describing

- Number of subjects needed

- How subjects will be selected

- Measurements to be made

-Iterative process

- Consultations with mentors/colleagues/literature

-Focus on : single primary question in designing and implementing the study 


\section{Suggested Exercises at Home}

-Look for research papers in your area of interest

-Assess if the RQ is explicitly stated

- Use the FINER criteria and the PICOT framework to critic the merits of the RQ

-Write your ideal research Qn (feasibility aside(:) for now)

- Use the FINER/PICOT to refine your Qn

-Share with your colleagues/mentors

-Visit the NIH RePORT to find funded research projec for your topic of interest 


\section{Resources}

- CIRHT research guide

- https://cirht.med.umich.edu/resources/

-NIH webpage

- Research Portfolio Online Reporting Tools (RePORT)

- https://report.nih.gov/ 
Thank You

Questions? 